\title{
Design, Synthesis, and Cytotoxic Activity of Michael Acceptors and Enol Esters in the Benzo[b]acronycine Series
}

\author{
Huong Doan Thi Mai,${ }^{a}$ Thomas Gaslonde, ${ }^{a}$ Sylvie Michel,${ }^{a}$ Michel Koch, ${ }^{a}$ François Tillequin,,$* a$ \\ Christian Bailly, ${ }^{b}$ Marie-Hélène David-Cordonnier, ${ }^{b}$ Bruno Pfeiffer, ${ }^{c}$ Stéphane Léonce, ${ }^{c}$ and \\ Alain PIERRÉ ${ }^{c}$ \\ ${ }^{a}$ Laboratoire de Pharmacognosie de l'Université René Descartes, U.M.R./C.N.R.S. n`8638, Faculté des Sciences \\ Pharmaceutiques et Biologiques; 4, Avenue de l'Observatoire, F-75006 Paris, France: ${ }^{b}$ INSERM U-524 et Laboratoire de \\ Pharmacologie Antitumorale du Centre Oscar Lambret, IRCL; 59045 Lille Cedex, France: and ${ }^{c}$ Institut de Recherches \\ Servier, Division Recherche Cancérologie; 125 Chemin de Ronde, 78290 Croissy sur Seine, France. \\ Received January 31, 2005; accepted April 18, 2005
}

\begin{abstract}
A series of 2-acyl-6-methoxy-3,3,14-trimethyl-3,14-dihydro-7H-benzo[b]pyrano[3,2- $h]$ acridin-7-ones (4-6) was prepared by treatment of 6-methoxy-3,3,14-trimethyl-3,14-dihydro-7H-benzo[b]pyrano[3,2- $h]$ acridin-7-one (3) with an excess of an appropriate acyl chloride in the presence of aluminum chloride. Treatment of ( \pm )-cis-1hydroxy-2-acyloxy-6-methoxy-3,3,14-trimethyl-1,2,3,14-tetrahydro-7H-benzo[b]pyrano[3,2- $h]$ acridin-7-ones $(9,10)$ or ( \pm )-cis-1,2-diacyloxy-6-methoxy-3,3,14-trimethyl-1,2,3,14-tetrahydro-7H-benzo[b]pyrano[3,2-h]acridin-7-ones $(2,11)$ with hydrochloric acid gave the corresponding 2-acyloxy-6-methoxy-3,3,14-trimethyl-3,14-dihydro-7Hbenzo[b]pyrano[3,2- $h$ ]acridin-7-ones, exemplified by acetate 7 and butyrate 8 . None of the Michael acceptors $4-$ 6 showed significant antiproliferative activity. Enol esters 7 and 8 were markedly cytotoxic toward L1210 leukemia cells, with $\mathrm{IC}_{50}$ values within the same range of magnitude as $( \pm)$-cis-1,2-diacetoxy-6-methoxy-3,3,14trimethyl-1,2,3,14-tetrahydro-7H-benzo[b]pyrano[3,2-h]acridin-7-one (S23906-1), currently under phase I clinical trials. In contrast with S23906-1, enol esters 7 and 8 were not reactive toward purified DNA.
\end{abstract}

Key words acronycine; benzo[b]acronycine; cytotoxicity

The pyranoacridone alkaloid acronycine (1), originally isolated from Acronychia baueri Sснотт (Rutaceae) ${ }^{1-3)}$ has shown antitumor properties in a panel of murine solid tumor models, including S-180 and AKR sarcomas, X-5563 myeloma, S-115 carcinoma, and S-91 melanoma. ${ }^{4,5)}$ However, its moderate potency and poor solubility in aqueous solvents severely hampered the subsequent clinical trials, which were rapidly discontinued, due to modest therapeutic effects and dose-limiting gastrointestinal toxicity after oral administration. ${ }^{6}$ Consequently, the development of structural analogues with increased potency and/or better water solubility was highly desirable.

Our efforts toward the obtainment of more potent derivatives were guided by a hypothesis of bioactivation of the 1,2double bond of acronycine into the corresponding epoxide in vivo. ${ }^{7)}$ Significant improvements in terms of solubility and potency were obtained with derivatives modified in the pyran ring, which had a similar reactivity toward nucleophilic agents as acronycine epoxide, but an improved chemical stability. Such compounds are exemplified by diesters of cis1,2-dihydroxy-1,2-dihydroacronycine ${ }^{8)}$ and diesters of cis1,2-dihydroxy-1,2-dihydrobenzo[b]acronycine $\quad(( \pm)$-cis-1,2dihydroxy-6-methoxy-3,3,14-trimethyl-1,2,3,14-tetrahydro$7 H$-benzo[ $b]$ pyrano[3,2- $h]$ acridin-7-one). ${ }^{9)}$ Representatives of this latter series are considered as valuable drug candidates for clinical studies. ${ }^{10)}$ For instance, diacetate 2 , currently de-

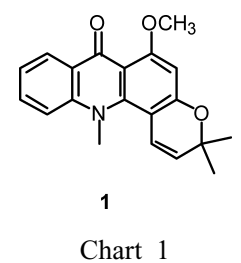

veloped under the code S23906-1 is currently under phase I clinical trials. Their mechanism of action implies alkylation of the 2-amino group of DNA guanine residues by the carbocation resulting from the elimination of the ester leaving group at position 1 of the drug. ${ }^{11-14)}$

In the course of the exploration of the structure activity relationships in the acronycine series, ${ }^{15)}$ 2-nitroacronycine, although shown to be toxic in vivo, demonstrated remarkably high potency when tested against a battery of cultured mammalian cells in vitro. ${ }^{4,16,17)}$ In this context, Michael acceptors in the benzo[b]acronycine series, possessing a 1,2-double bond and substituted at position 2 by an acyl group appeared as possible new drug candidates able to undergo additions at position 1 onto intracellular nuclophilic targets. Additionally, enol esters at position 2 were also worth exploring for comparison purposes. We describe here the synthesis and biological activities of various 2-acyl- and 2-acyloxy-6-methoxy3,3,14-trimethyl-3,14-dihydro-7H-benzo[b]pyrano[3,2h] acridin-7-ones.

\section{Chemistry}

Treatment of 6-methoxy-3,3,14-trimethyl-3,14-dihydro$7 H$-benzo[b]pyrano[3,2- $h]$ acridin-7-one (3) with an excess of an appropriate acyl chloride in the presence of aluminum chloride in dichloromethane afforded the corresponding 2acyl-6-methoxy-3,3,14-trimethyl-3,14-dihydro-7 $H$-benzo$[b]$ pyrano[3,2- $h]$ acridin-7-ones. Following this procedure, the desired 2-acetyl-6-methoxy-3,3,14-trimethyl-3,14-dihydro-7H-benzo[b]pyrano[3,2- $h$ ]acridin-7-one (4), 2-butyryl-6methoxy-3,3,14-trimethyl-3,14-dihydro-7H-benzo[b]pyrano[3,2-h]acridin-7-one (5), and 2-benzoyl-6-methoxy3,3,14-trimethyl-3,14-dihydro-7H-benzo[b]pyrano[3,2$h$ ]acridin-7-one (6) were prepared in 96,54 , and $42 \%$ yield, respectively. 


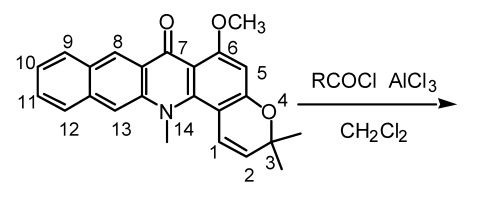

3

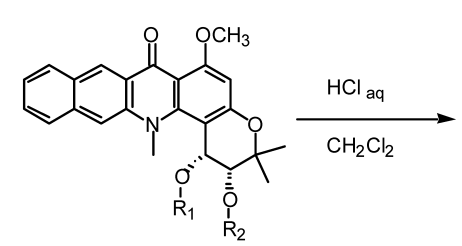

(士) $2 \mathrm{R}_{1}=\mathrm{R}_{2}=\mathrm{CH}_{3} \mathrm{CO}$

(士) $11 \mathrm{R}_{1}=\mathrm{R}_{2}=\mathrm{CH}_{3} \mathrm{CH}_{2} \mathrm{CH}_{2} \mathrm{CO}$

(士) $9 \quad \mathrm{R}_{1}=\mathrm{H}, \mathrm{R}_{2}=\mathrm{CH}_{3} \mathrm{CO}$

( \pm ) $10 \mathrm{R}_{1}=\mathrm{H}, \mathrm{R}_{2}=\mathrm{CH}_{3} \mathrm{CH}_{2} \mathrm{CH}_{2} \mathrm{CO}$

Chart 2

Enol esters at position 2, exemplified by acetate 7 and butyrate 8, were obtained in moderate 40 and $31 \%$ yield, respectively, by dehydration of their corresponding ( \pm )-cis-1hydroxy-2-acyloxy-6-methoxy-3,3,14-trimethyl-1,2,3,14tetrahydro-7H-benzo[ $b]$ pyrano[3,2- $h]$ acridin-7-ones 9 and $\mathbf{1 0}$ with hydrochloric acid in dichloromethane. When the same reaction was applied to $( \pm)$-cis-1,2-diacyloxy-6-methoxy-3,3,14-trimethyl-1,2,3,14-tetrahydro-7H-benzo[b]pyrano[3,2h]acridin-7-ones 2 and 11, enol esters 7 and 8 were also obtained, but in lower yields $(19,12 \%$, respectively) than when the monoesters $\mathbf{9}$ and $\mathbf{1 0}$ were used as starting materials.

\section{Pharmacology}

The study of the biological properties of the new 2-acylbenzo $[b]$ acronycines $\mathbf{4}, \mathbf{5}$, and $\mathbf{6}$, and of the enol esters $\mathbf{7}$ and 8 was carried out in vitro in the L1210 murine leukemia cell line. The results $\left(\mathrm{IC}_{50}\right.$ values) are reported in Table 1 . None of the Michael acceptors $\mathbf{4 - 6}$ showed significant antiproliferative activity. In contrast, enol esters $\mathbf{7}$ and $\mathbf{8}$ were markedly cytotoxic, with $\mathrm{IC}_{50}$ values within the same range of magnitude as diacetate $\mathbf{2}$, currently under phase I clinical trials.

The perturbation of the cell cycle induced by the active enol esters $\mathbf{7}$ and $\mathbf{8}$ was studied in the same cell line. Gel retardation experiments with DNA fragments were also performed for these two compounds, in comparison with diacetate $\mathbf{2}$ previously shown to alkylate purified DNA at the N-2 of guanine residues. ${ }^{11,12)}$ The results are summarized in Table 2. As previously established, diacetate $\mathbf{2}$ induced a partially reversible accumulation in the $\mathrm{G} 2+\mathrm{M}$ phases of the cell cycle at low concentration and an irreversible arrest in the $\mathrm{S}$ phase at higher concentration, whereas accumulation in the G1 phase was observed with enol acetate 7. No alkylation of purified DNA was observed with both compounds 7 and 8 . At the cellular level, the mechanism of action of the compound $\mathbf{8}$ is different from that of $\mathbf{2}$. Indeed, compound $\mathbf{8}$ did not induce an $\mathrm{S}$ phase accumulation, which is a common characteristic of all the derivatives acting as compound $\mathbf{2}$. Compound $\mathbf{8}$ only induced a partial accumulation in the $\mathrm{G} 2+\mathrm{M}$ phases. The mechanism of action of compound 7 is
Table 1. Inhibition of L1210 Cell Proliferation by Compounds $\mathbf{4}-\mathbf{8}$ in Comparison with Benzo[b]acronycine (3) and S23906-1 (2)

\begin{tabular}{lccccccc}
\hline \hline Compound & $\mathbf{2}$ & $\mathbf{3}$ & $\mathbf{4}$ & $\mathbf{5}$ & $\mathbf{6}$ & $\mathbf{7}$ & $\mathbf{8}$ \\
\hline $\mathrm{IC}_{50}(\mu \mathrm{M})$ & 0.8 & 15 & 20 & 30 & $>50$ & 0.75 & 1.8 \\
\hline
\end{tabular}

Table 2. Cell Cycle Perturbation Induced in L1210 Cell Line and in Vitro DNA Alkylation Induced by Compounds $\mathbf{7}$ and $\mathbf{8}$ in Comparison with S23906-1 (2)

\begin{tabular}{|c|c|c|c|}
\hline Compound & 2 & 7 & 8 \\
\hline Cell cycle perturbation & $\begin{array}{c}72 \% \mathrm{G} 2+\mathrm{M} \\
(1 \mu \mathrm{M}) \\
73 \% \mathrm{~S} \\
(5 \mu \mathrm{M})\end{array}$ & $\begin{array}{l}55 \% \mathrm{G} 1 \\
(20 \mu \mathrm{M})\end{array}$ & $\begin{array}{c}51 \% \mathrm{G} 2+\mathrm{M} \\
(20 \mu \mathrm{M})\end{array}$ \\
\hline In vitro DNA Alkylation & ++ & 0 & 0 \\
\hline
\end{tabular}

The capacity of the tested compounds to form complexes with purified DNA was investigated by gel shift assay. Symbol ++ refers to strong alkylation, whereas 0 means no alkylation at all.

clearly different from that of compounds $\mathbf{2}$ and $\mathbf{8}$, as shown by a completely different perturbation of the cell cycle, i.e. slowing down during the G1 phase.

\section{Results and Discussion}

Considering the structure activity relationships in the benzo[ $b]$ acronycine series, it appears that compounds bearing an acyl substituent at position 2, initially considered as Michael acceptors able to undergo additions to nucleophilic cellular targets, were devoid of cytotoxic activity. From a chemical point of view, this result should be correlated with the high delocalization of the electrons in the benzo[b]acronycine chromophore.

Enol esters at position 2, exemplified by $\mathbf{7}$ and $\mathbf{8}$, displayed cytotoxic activities within the same range of magnitude as ( \pm )-cis-1,2-diacetoxy-1,2-dihydro benzo[b]acronycine. Nevertheless, the mechanism of their action at the molecular level differs from that of $\mathbf{2}$. In agreement with this statement, both enol esters $\mathbf{7}$ and $\mathbf{8}$ did not bind to purified DNA.

\section{Experimental}

Chemistry The melting points were determined on a Leica VM apparatus and are not corrected. IR spectra $\left(v_{\max }\right.$ in $\left.\mathrm{cm}^{-1}\right)$ were obtained on a Perkin-Elmer 257 instrument. UV spectra $\left(\lambda_{\max }\right.$ in $\left.\mathrm{nm}\right)$ were determined in spectroscopic-grade $\mathrm{MeOH}$ on a Beckman Model 34 spectrophotometer. ${ }^{1} \mathrm{H}$ $\operatorname{NMR}(\delta[\mathrm{ppm}], J[\mathrm{~Hz}])$ and ${ }^{13} \mathrm{C}-\mathrm{NMR}$ spectra were recorded at 400 and $100 \mathrm{MHz}$ respectively, using a Bruker Avance 400 spectrometer. When necessary, the signals were unambiguously assigned by $2 \mathrm{D}$ NMR techniques: ${ }^{1} \mathrm{H}-{ }^{1} \mathrm{H}$ COSY, ${ }^{1} \mathrm{H}-{ }^{1} \mathrm{H}$ NOESY, ${ }^{13} \mathrm{C}-{ }^{1} \mathrm{H}$ HMQC, and ${ }^{13} \mathrm{C}-{ }^{1} \mathrm{H}$ HMBC. These experiments were performed using standard Bruker microprograms. Mass spectra were recorded with a Nermag R-10-10C spectrometer using electron impact ionization (EI-MS; $70 \mathrm{eV}$ ) technique. Flash column chromatographies were performed using silica gel 60 Merck $(35-70 \mathrm{~mm})$ with an overpressure of 300 mbar.

2-Acetyl-6-methoxy-3,3,14-trimethyl-3,14-dihydro-7H-benzo [b]pyrano[3,2- $\boldsymbol{h}$ ]acridin-7-one (4) Acetyl chloride $(59 \mu \mathrm{l}, 0.824 \mathrm{mmol})$ and aluminium chloride $(108 \mathrm{mg}, 0.673 \mathrm{mmol})$ were added to a solution of 6methoxy-3,3,14-trimethyl-3,14-dihydro-7H-benzo[ $b]$ pyrano[3,2-h]acridin-7one (3) $(50 \mathrm{mg}, 0.1374 \mathrm{mmol})$ in $\mathrm{CH}_{2} \mathrm{Cl}_{2}(2 \mathrm{ml})$ at $0{ }^{\circ} \mathrm{C}$. The reaction mixture was stirred at $20^{\circ} \mathrm{C}$ for $3 \mathrm{~h}$ and poured onto $10 \%$ aqueous $\mathrm{HCl}$ solution $(100 \mathrm{ml})$ at $0{ }^{\circ} \mathrm{C}$. The mixture was extracted with $\mathrm{CH}_{2} \mathrm{Cl}_{2}(3 \times 100 \mathrm{ml})$. The combined organic layer was washed with saturated $\mathrm{Na}_{2} \mathrm{CO}_{3}$ aqueous solution $(100 \mathrm{ml})$, water $(2 \times 100 \mathrm{ml})$, dried over anhydrous $\mathrm{Na}_{2} \mathrm{SO}_{4}$, and evaporated under reduced pressure. Flash chromatography (solvent: $\mathrm{CH}_{2} \mathrm{Cl}_{2}$, then $\mathrm{CH}_{2} \mathrm{Cl}_{2} / \mathrm{MeOH} 99: 1$ to $\left.95: 5\right)$ gave $4(54 \mathrm{mg}, 96 \%)$ as yellow needles, $\mathrm{mp}$ 
$277^{\circ} \mathrm{C}(\mathrm{EtOH}) . \mathrm{IR}(\mathrm{KBr}) \mathrm{cm}^{-1}: 3050,2973,2940,1647,1602,1559,1488$, $1459,1405,1364,1238,1209,1123,1087,809,735$. UV $\lambda_{\max }(\mathrm{MeOH}) \mathrm{nm}$ ( $\log \varepsilon$ ): 238 (4.36), 271 (4.76), 339 (4.58), 440 (3.85). ${ }^{1} \mathrm{H}-\mathrm{NMR}(400 \mathrm{MHz}$, $\left.\mathrm{CDCl}_{3}\right) \delta: 8.94(1 \mathrm{H}, \mathrm{s}, \mathrm{C} 8-\mathrm{H}), 8.05(1 \mathrm{H}, \mathrm{dd}, J=9,1 \mathrm{~Hz}, \mathrm{C} 9-\mathrm{H}), 7.89(1 \mathrm{H}$, dd, $J=9,1 \mathrm{~Hz}, \mathrm{C} 12-\mathrm{H}), 7.73(1 \mathrm{H}, \mathrm{s}, \mathrm{C} 13-\mathrm{H}), 7.59(1 \mathrm{H}$, ddd, $J=9,8,1 \mathrm{~Hz}$, C11-H), 7.55 (1H, s, C1-H), 7.47 (1H, ddd, $J=9,8,1 \mathrm{~Hz}, \mathrm{C} 10-\mathrm{H}), 6.29$ (1H, s, C5-H), $4.03\left(3 \mathrm{H}, \mathrm{s}, \mathrm{O}-\mathrm{C}_{3}\right), 3.99\left(3 \mathrm{H}, \mathrm{s}, \mathrm{N}-\mathrm{C}_{3}\right), 2.47(3 \mathrm{H}, \mathrm{s}, \mathrm{C} 2-$ $\left.\mathrm{COCH}_{3}\right), 1.72\left(6 \mathrm{H}, \mathrm{s}, \mathrm{C} 3-\left(\mathrm{C}_{3}\right)_{2}\right) .{ }^{13} \mathrm{C}-\mathrm{NMR}\left(75 \mathrm{MHz}, \mathrm{CDCl}_{3}\right) \delta: 195.2$ (C2-ㅡㅇ-), 177.5 (C-7), 165.9 (C-6), 161.3 (C-4a), 148.4 (C-14a), 142.1 (C13a), 135.7 (C-12a), 132.6 (C-2), 131.5 (C-1), 129.6 (C-9), 129.0 (C-8a), 128.5 (C-11), 128.3 (C-8), 126.8 (C-12), 125.4 (C-7a), 124.9 (C-10), 112.4 (C-13), 109.6 (C-6a), 102.4 (C-14b), 93.7 (C-5), 79.9 (C-3), $56.6\left(\mathrm{OCH}_{3}\right)$, $45.4\left(\mathrm{NCH}_{3}\right), 26.6\left(\mathrm{C} 2-\mathrm{COCH}{ }_{3}\right), 25.9\left(\mathrm{C} 3\left(\underline{\mathrm{CH}}_{3}\right)_{2}\right)$. EI-MS m/z: $413[\mathrm{M}]^{+}$, $398\left[\mathrm{M}-\mathrm{CH}_{3}\right]^{+}$. Anal. Calcd for $\mathrm{C}_{26} \mathrm{H}_{23} \mathrm{NO}_{4}: \mathrm{C}, 75.53 ; \mathrm{H}, 5.61 ; \mathrm{N}, 3.39$. Found: C, 75.22; H, 5.67; N, 3.46.

2-Butyryl-6-methoxy-3,3,14-trimethyl-3,14-dihydro-7H-benzo[b]pyrano[3,2-h]acridin-7-one (5) Compound 5 was obtained from 3 $(50 \mathrm{mg}, 0.137 \mathrm{mmol})$ under conditions similar with those described for the preparation of 4 , using butyryl chloride $(84 \mu 1,0.808 \mathrm{mmol})$ instead of acetyl chloride. Purification by flash chromatography (solvent: $\mathrm{CH}_{2} \mathrm{Cl}_{2}$, then $\mathrm{CH}_{2} \mathrm{Cl}_{2} / \mathrm{MeOH} 99: 1$ to $\left.94: 6\right)$ gave $5(32 \mathrm{mg}, 54 \%)$ as pale yellow needles, $\mathrm{mp} 302^{\circ} \mathrm{C}$ (acetone/hexane $7: 3$ ). IR $(\mathrm{KBr}) \mathrm{cm}^{-1}: 3050,2966,2870,1636$, 1592, 1577, 1563, 1489, 1462, 1404, 1360, 1246, 1211, 1172, 1125, 1087, 1028, 804, 738. UV $\lambda_{\max }(\mathrm{MeOH}) \mathrm{nm}(\log \varepsilon): 237$ (4.40), 271 (4.79), 339 (4.61), 442 (3.87). ${ }^{1} \mathrm{H}-\mathrm{NMR}\left(400 \mathrm{MHz}, \mathrm{CDCl}_{3}\right) \delta$ : $8.94(1 \mathrm{H}, \mathrm{s}, \mathrm{C} 8-\mathrm{H}), 8.05$ $(1 \mathrm{H}, \mathrm{dd}, J=9,1 \mathrm{~Hz}, \mathrm{C} 9-\mathrm{H}), 7.89(1 \mathrm{H}, \mathrm{dd}, J=9,1 \mathrm{~Hz}, \mathrm{C} 12-\mathrm{H}), 7.74(1 \mathrm{H}, \mathrm{s}$, C13-H), 7.57 (1H, ddd, $J=9,8,1 \mathrm{~Hz}, \mathrm{C} 11-\mathrm{H}), 7.50(1 \mathrm{H}, \mathrm{s}, \mathrm{C} 1-\mathrm{H}), 7.45(1 \mathrm{H}$ ddd, $J=9,8,1 \mathrm{~Hz}, \mathrm{C} 10-\mathrm{H}), 6.29(1 \mathrm{H}, \mathrm{s}, \mathrm{C} 5-\mathrm{H}), 4.04\left(3 \mathrm{H}, \mathrm{s}, \mathrm{O}-\mathrm{CH}_{3}\right), 3.97$ $\left(3 \mathrm{H}, \mathrm{s}, \mathrm{N}-\mathrm{CH}_{3}\right), 2.76\left(2 \mathrm{H}, \mathrm{t}, J=7.5 \mathrm{~Hz}, \mathrm{C} 2-\mathrm{COCH}_{2} \mathrm{CH}_{2} \mathrm{CH}_{3}\right), 1.75(2 \mathrm{H}, \mathrm{m}$, $\left.\mathrm{C} 2-\mathrm{COCH}_{2} \mathrm{CH}_{2} \mathrm{CH}_{3}\right), 1.72\left(6 \mathrm{H}, \mathrm{s}, \mathrm{C} 3-\left(\mathrm{CH}_{3}\right)_{2}\right), 1.02(3 \mathrm{H}, \mathrm{t}, J=7.5 \mathrm{~Hz}, \mathrm{C} 2-$ $\left.\mathrm{COCH}_{2} \mathrm{CH}_{2} \mathrm{CH}_{3}\right) .{ }^{13} \mathrm{C}-\mathrm{NMR}\left(75 \mathrm{MHz}, \mathrm{CDCl}_{3}\right) \delta$ : 198.1 (C2-CO-), 177.6 (C-7), 165.7 (C-6), $161.2(\mathrm{C}-4 \mathrm{a}), 148.8$ (C-14a), 141.5 (C-13a), 135.7 (C12a), 131.6 (C-2), 131.1 (C-1), 129.7 (C-9), 128.9 (C-8a), 128.5 (C-11), 128.4 (C-8), 126.8 (C-12), 125.4 (C-7a), 124.9 (C-10), 112.4 (C-13), 110.0 (C-6a), 102.1 (C-14b), 93.7 (C-5), 79.9 (C-3), $56.6\left(\mathrm{OCH}_{3}\right), 45.4\left(\mathrm{NC}_{3}\right)$, $40.3\left(\mathrm{C} 2-\mathrm{COCH}_{2} \mathrm{CH}_{2} \mathrm{CH}_{3}\right), 25.9\left(\mathrm{C} 3\left(\mathrm{CH}_{3}\right)_{2}\right), 18.6\left(\mathrm{C}_{2}-\mathrm{COCH}_{2} \mathrm{CH}_{2} \mathrm{CH}_{3}\right)$, $13.9\left(\mathrm{C} 2-\mathrm{COCH}_{2} \mathrm{CH}_{2} \mathrm{CH}_{3}\right)$. EI-MS m/z: $441[\mathrm{M}]^{+}, 426\left[\mathrm{M}-\mathrm{CH}_{3}\right]^{+}, 412$ $\left[\mathrm{M}-\mathrm{C}_{2} \mathrm{H}_{5}\right]^{+}$. Anal. Calcd for $\mathrm{C}_{28} \mathrm{H}_{27} \mathrm{NO}_{4}: \mathrm{C}, 76.17 ; \mathrm{H}, 6.16 ; \mathrm{N}, 3.17$. Found: C, 76.21; H, 6.07; N, 3.26.

2-Benzoyl-6-methoxy-3,3,14-trimethyl-3,14-dihydro-7H-benzo[b]pyrano[3,2-h]acridin-7-one (6) Compound 6 was obtained from 3 $(50 \mathrm{mg}, 0.137 \mathrm{mmol})$ under conditions similar with those described for the preparation of 4 , using benzoyl chloride $(384 \mu 1,3.23 \mathrm{mmol})$ instead of acetyl chloride. Purification by flash chromatography (solvent: $\mathrm{CH}_{2} \mathrm{Cl}_{2}$, then $\mathrm{CH}_{2} \mathrm{Cl}_{2} / \mathrm{MeOH} 99: 1$ to $\left.94: 6\right)$ gave $6(27 \mathrm{mg}, 42 \%)$ as an orange amorphous solid. IR (KBr) $\mathrm{cm}^{-1}$ : 3050, 2966, 2929, 1632, 1510, 1585, 1559, 1489 , $1459,1403,1360,1246,1213,1135,1084,1025,805,729$. UV $\lambda_{\max }$ $(\mathrm{MeOH}) \mathrm{nm}(\log \varepsilon): 240$ (4.37), 271 (4.62), 343 (4.42), 437 (4.14). ${ }^{1} \mathrm{H}-\mathrm{NMR}$ $\left(400 \mathrm{MHz} \mathrm{CDCl}_{3}\right) \delta: 8.94(1 \mathrm{H}, \mathrm{s}, \mathrm{C} 8-\mathrm{H}), 8.03(1 \mathrm{H}, \mathrm{d}, J=9 \mathrm{~Hz}, \mathrm{C} 9-\mathrm{H}), 7.80$ (3H, m, C12-H, C2' $\left.-\mathrm{H}, \mathrm{C}^{\prime}-\mathrm{H}\right), 7.57$ (3H, m, C13-H, C11-H, C4'-H), 7.48 $\left(3 \mathrm{H}, \mathrm{m}, \mathrm{C} 10-\mathrm{H}, \mathrm{C}^{\prime}-\mathrm{H}, \mathrm{C}^{\prime}-\mathrm{H}\right), 7.10(1 \mathrm{H}, \mathrm{s}, \mathrm{C} 1-\mathrm{H}), 6.36(1 \mathrm{H}, \mathrm{s}, \mathrm{C} 5-\mathrm{H}), 4.07$ $\left(3 \mathrm{H}, \mathrm{s}, \mathrm{O}-\mathrm{CH}_{3}\right), 3.73\left(3 \mathrm{H}, \mathrm{s}, \mathrm{N}-\mathrm{CH}_{3}\right), 1.82\left(6 \mathrm{H}, \mathrm{s}, \mathrm{C} 3\left(\mathrm{CH}_{3}\right)_{2}\right) .{ }^{13} \mathrm{C}-\mathrm{NMR}$ $\left(75 \mathrm{MHz} \mathrm{CDCl}_{3}\right) \delta: 193.9(\mathrm{C} 2-\mathrm{CO}-), 178.0(\mathrm{C}-7), 165.6(\mathrm{C}-6), 161.3(\mathrm{C}-$ 4a), 148.6 (C-14a), 140.9 (C-13a), 137.9 (C-12a), 135.6 (C-2), 134.0 (C-1) $132.4\left(\mathrm{C}-4^{\prime}\right), 130.5\left(\mathrm{C}-1^{\prime}\right), 129.4\left(\mathrm{C}-2^{\prime}+\mathrm{C}^{\prime} 6^{\prime}\right), 129.3$ (C-9), 128.7 (C-8a), $128.4\left(\mathrm{C}-11+\mathrm{C}-3^{\prime}+\mathrm{C}^{\prime} 5^{\prime}\right), 128.0(\mathrm{C}-8), 126.7$ (C-12), 124.9 (C-10), 124.7 (C-7a), 112.4 (C-13), 109.1 (C-6a), 102.2 (C-14b), 93.6 (C-5), 79.8 (C-3), $56.2\left(\mathrm{OCH}_{3}\right), 44.8\left(\mathrm{NCH}_{3}\right), 25.2\left(\mathrm{C} 3\left(\underline{\mathrm{CH}}_{3}\right)_{2}\right)$. EI-MS m/z: $475[\mathrm{M}]^{+\cdot}, 460$ $\left[\mathrm{M}-\mathrm{CH}_{3}\right]^{+}$. Anal. Calcd for $\mathrm{C}_{31} \mathrm{H}_{25} \mathrm{NO}_{4}$ : C, 78.30; H, 5.30; N, 2.95. Found: C, 78.20; H, 5.37; N, 2.96 .

2-Acetoxy-6-methoxy-3,3,14-trimethyl-3,14-dihydro-7H-benzo [b]pyrano[3,2- $\boldsymbol{h}]$ acridin-7-one (7) Method a: Aqueous $\mathrm{HCl}$ solution $(10 \%$, $0.2 \mathrm{ml}$ ) was added to a solution of $( \pm)$-cis-2-acetoxy-1-hydroxy-6-methoxy3,3,14-trimethyl-1,2,3,14-tetrahydro-7H-benzo[b]pyrano[3,2- $h]$ acridin-7one (9) $(40 \mathrm{mg}, 0.082 \mathrm{mmol})$ in $\mathrm{CH}_{2} \mathrm{Cl}_{2}(2 \mathrm{ml})$. The reaction mixture was stirred for $4 \mathrm{~d}$ at $20^{\circ} \mathrm{C}$ and evaporated under reduced pressure. Flash chromatography (solvent: $\mathrm{CH}_{2} \mathrm{Cl}_{2}$, then $\mathrm{CH}_{2} \mathrm{Cl}_{2} / \mathrm{MeOH} 99: 1$ to $90: 10$ ) gave 7 (14 mg, 40\%) as orange needles, mp $165^{\circ} \mathrm{C}$ (acetone). IR (KBr) cm ${ }^{-1}: 3048$, 2971, 2931, 1765, 1647, 1620, 1586, 1569, 1492, 1461, 1397, 1333, 1231, $1194,1110,1086,1029,874,799$. UV $\lambda_{\text {max }}(\mathrm{MeOH}) \mathrm{nm}(\log \varepsilon): 240$ (4.37), 275 (4.72), 307 (4.70), 360 (3.93), 443 (3.71). ${ }^{1} \mathrm{H}-\mathrm{NMR}\left(400 \mathrm{MHz}, \mathrm{CDCl}_{3}\right)$ $\delta: 8.90(1 \mathrm{H}, \mathrm{s}, \mathrm{C} 8-\mathrm{H}), 8.02(1 \mathrm{H}, \mathrm{dd}, J=9,1 \mathrm{~Hz}, \mathrm{C} 9-\mathrm{H}), 7.87(1 \mathrm{H}, \mathrm{dd}, J=9$, $1 \mathrm{~Hz}, \mathrm{C} 12-\mathrm{H}), 7.65$ (1H, s, C13-H), 7.54 (1H, ddd, $J=9,8,1 \mathrm{~Hz}, \mathrm{C} 11-\mathrm{H})$,
$7.41(1 \mathrm{H}$, ddd, $J=9,8,1 \mathrm{~Hz}, \mathrm{C} 10-\mathrm{H}), 6.54(1 \mathrm{H}, \mathrm{s}, \mathrm{C} 1-\mathrm{H}), 6.35(1 \mathrm{H}, \mathrm{s}, \mathrm{C} 5-$ $\mathrm{H}), 4.01\left(3 \mathrm{H}, \mathrm{s}, \mathrm{O}-\mathrm{CH}_{3}\right), 3.95\left(3 \mathrm{H}, \mathrm{s}, \mathrm{N}-\mathrm{CH}_{3}\right), 2.29\left(3 \mathrm{H}, \mathrm{s}, \mathrm{C} 2-\mathrm{OCOC} \underline{H}_{3}\right)$, $1.59\left(6 \mathrm{H}, \mathrm{s}, \mathrm{C} 3-\left(\mathrm{CH}_{3}\right)_{2}\right) \cdot{ }^{13} \mathrm{C}-\mathrm{NMR}\left(75 \mathrm{MHz}, \mathrm{CDCl}_{3}\right) \delta: 178.2(\mathrm{C}-7), 169.2$ $\left(\mathrm{C} 2-\mathrm{OCOCH}_{3}\right), 163.0$ (C-6), 157.7 (C-4a), 150.1 (C-14a), 142.0 (C-2), 141.0 (C-13a), 135.8 (C-12a), 129.6 (C-9), 128.7 (C-8a), $128.2(\mathrm{C}-11+\mathrm{C}-8)$, 126.8 (C-12), 125.5 (C-7a), 124.5 (C-10), 112.5 (C-6a), 111.9 (C-13), 109.9 (C-5), 102.3 (C-14b), 93.8 (C-1), 78.0 (C-3), $56.4\left(\mathrm{OCH}_{3}\right), 44.7\left(\mathrm{NCH}_{3}\right)$, $24.1\left(\mathrm{C} 3\left(\mathrm{CH}_{3}\right)_{2}\right), 21.1 \quad\left(\mathrm{C} 2-\mathrm{OCOCH}_{3}\right) . \quad$ EI-MS $m / z: 429 \quad[\mathrm{M}]^{+\cdot}, 387$ $\left[\mathrm{M}-\mathrm{COCH}_{2}\right]^{+\cdot}$. Anal. Calcd for $\mathrm{C}_{26} \mathrm{H}_{23} \mathrm{NO}_{5}$ : C, 72.71; H, 5.40; N, 3.26 . Found: C, 77.81; H, 5.45; N, 3.21.

Method b: The same reaction applied to ( \pm )-cis-1,2-diacetoxy-6methoxy-3,3,14-trimethyl-1,2,3,14-tetrahydro-7H-benzo[b]pyrano[3,2h] acridin-7-one (2) (40 mg, $0.0818 \mathrm{mmol}$ ) afforded 7 (11 mg, 31\%).

2-Butyroxy-6-methoxy-3,3,14-trimethyl-3,14-dihydro-7H-benzo[b]pyrano[3,2-h]acridin-7-one (8) Compound 8 was obtained from ( \pm )cis-2-butyryloxy-1-hydroxy-6-methoxy-3,3,14-trimethyl-1,2,3,14-tetrahydro-7H-benzo[b]pyrano[3,2- $h$ ]acridin-7-one (10) (method a) or ( \pm )-cis-1,2dibutyryloxy-6-methoxy-3,3,14-trimethyl-1,2,3,14-tetrahydro-7 Hbenzo[b]pyrano[3,2- $h$ ]acridin-7-one (11) (method b) under conditions similar with those described for the preparation of 7 . Purification by flash chromatography (solvent: $\mathrm{CH}_{2} \mathrm{Cl}_{2}$, then $\mathrm{CH}_{2} \mathrm{Cl}_{2} / \mathrm{MeOH} 99: 1$ to $95: 5$ ) gave 5 in 19 and $12 \%$ yield, respectively, as orange needles, mp $180{ }^{\circ} \mathrm{C}\left(\mathrm{CH}_{2} \mathrm{Cl}_{2}\right)$. IR $(\mathrm{KBr}) \mathrm{cm}^{-1}:$ 3052, 2960, 1756, 1649, 1618, 1587, 1566, 1494, 1460, 1397, $1332,1211,1138,1108,1084,1025,872,811,745$. UV $\lambda_{\max }(\mathrm{MeOH}) \mathrm{nm}$ ( $\log \varepsilon$ ): 239 (4.43), 275 (4.77), 307 (4.72), 360 (3.95), 440 (3.77). ${ }^{1} \mathrm{H}-\mathrm{NMR}$ $\left(400 \mathrm{MHz}, \mathrm{CDCl}_{3}\right) \delta: 8.90(1 \mathrm{H}, \mathrm{s}, \mathrm{C} 8-\mathrm{H}), 8.01(1 \mathrm{H}, \mathrm{dd}, J=9,1 \mathrm{~Hz}, \mathrm{C} 9-\mathrm{H})$, $7.84(1 \mathrm{H}, \mathrm{dd}, J=9,1 \mathrm{~Hz}, \mathrm{C} 12-\mathrm{H}), 7.65(1 \mathrm{H}, \mathrm{s}, \mathrm{C} 13-\mathrm{H}), 7.53(1 \mathrm{H}$, ddd, $J=9$, $8,1 \mathrm{~Hz}, \mathrm{C} 11-\mathrm{H}), 7.40(1 \mathrm{H}, \mathrm{ddd}, J=9,8,1 \mathrm{~Hz}, \mathrm{C} 10-\mathrm{H}), 6.55(1 \mathrm{H}, \mathrm{s}, \mathrm{C} 1-\mathrm{H})$ $6.32(1 \mathrm{H}, \mathrm{s}, \mathrm{C} 5-\mathrm{H}), 4.00\left(3 \mathrm{H}, \mathrm{s}, \mathrm{O}-\mathrm{CH}_{3}\right), 3.94\left(3 \mathrm{H}, \mathrm{s}, \mathrm{N}-\mathrm{CH}_{3}\right), 2.52(2 \mathrm{H}, \mathrm{t}$, $\left.J=7.5 \mathrm{~Hz}, \mathrm{C} 2-\mathrm{COCH}_{2} \mathrm{CH}_{2} \mathrm{CH}_{3}\right), 1.79\left(2 \mathrm{H}, \mathrm{m}, \mathrm{C} 2-\mathrm{COCH}_{2} \mathrm{CH}_{2} \mathrm{CH}_{3}\right), 1.59$ $\left(6 \mathrm{H}, \mathrm{s}, \mathrm{C} 3-\left(\mathrm{CH}_{3}\right)_{2}\right), 1.07\left(3 \mathrm{H}, \mathrm{t}, J=7.5 \mathrm{~Hz}, \mathrm{C} 2-\mathrm{COCH}_{2} \mathrm{CH}_{2} \mathrm{CH}_{3}\right) .{ }^{13} \mathrm{C}-\mathrm{NMR}$ $\left(75 \mathrm{MHz}, \mathrm{CDCl}_{3}\right) \delta: 178.0(\mathrm{C}-7), 172.0(\mathrm{C} 2-\mathrm{OCO}), 163.0(\mathrm{C}-6), 157.6(\mathrm{C}-$ 4a), 150.3 (C-14a), 147.5 (C-2), 141.0 (C-13a), 135.8 (C-12a), 129.6 (C-9), 128.7 (C-8a), 128.2 (C-11+C-8), 126.7 (C-12), 125.7 (C-7a), 124.5 (C-10), 111.9 (C-13), 110.1 (C-6a), 109.7 (C-5), 102.4 (C-14b), 98.8 (C-1), 77.2 (C3), $56.4\left(\mathrm{OCH}_{3}\right), \quad 44.6\left(\mathrm{NCH}_{3}\right), \quad 36.3 \quad\left(\mathrm{C} 2-\mathrm{OCOCH}_{2} \mathrm{CH}_{2} \mathrm{CH}_{3}\right), \quad 24.1$ $\left(\mathrm{C} 3\left(\underline{\mathrm{CH}}_{3}\right)_{2}\right), 18.4\left(\mathrm{C} 2-\mathrm{OCOCH}_{2} \underline{\mathrm{CH}}_{2} \mathrm{CH}_{3}\right), 13.7\left(\mathrm{C} 2-\mathrm{OCOCH}_{2} \mathrm{CH}_{2} \underline{\mathrm{CH}} \mathrm{H}_{3}\right)$. EIMS $m / z: 457[\mathrm{M}]^{+}$, $387\left[\mathrm{M}-\mathrm{CO}\left(\mathrm{CH}_{2}\right)_{3}\right]^{+\cdot}$. Anal. Calcd for $\mathrm{C}_{28} \mathrm{H}_{27} \mathrm{NO}_{5}: \mathrm{C}$, 73.51; H, 5.95; N, 3.06. Found: C, 73.58; H, 6.01; N, 2.99.

Pharmacology. Cytotoxicity Murine leukemia L1210 cells from the American Type Culture Collection (Rockville Pike, MD, U.S.A.) were grown in RPMI medium 1640 supplemented with $10 \%$ fetal calf serum, $2 \mathrm{~mm}$ L-glutamine, penicillin $100 \mathrm{U} / \mathrm{ml}$, streptomycin $100 \mu \mathrm{g} / \mathrm{ml}$ and $10 \mathrm{~mm}$ HEPES buffer ( $\mathrm{pH}$ 7.4). The cytotoxicity was measured using the microculture tetrazolium assay essentially as described. ${ }^{16)}$ Cells were exposed for $48 \mathrm{~h}$ to nine graded concentrations in triplicate of the test drug. Results are expressed as $\mathrm{IC}_{50}$ values (mean, $n=3$ ), which are defined as the drug concentration inhibiting the absorbance by $50 \%$ with respect to that of untreated cells.

Cell Cycle Analysis For the cell cycle analysis, L1210 cells (5X $10^{5}$ cells $/ \mathrm{ml}$ ) were incubated for $21 \mathrm{~h}$ with various concentrations of drugs. Cells were then fixed with $70 \%$ ethanol (v/v), washed, and incubated in PBS containing RNAse $100 \mu \mathrm{g} / \mathrm{ml}$ and propidium iodide $50 \mu \mathrm{g} / \mathrm{ml}$ for $30 \mathrm{~min}$ at $20^{\circ} \mathrm{C}$. For each sample, 10000 cells were analyzed on an XLMCL flow cytometer (Beckman Coulter, France). Results are expressed as the percentage of cells arrested in the given phases of the cell cycle.

Binding to DNA: Gel Shift Studies A typical cross-linking reaction consisted of incubating $8 \mu \mathrm{l}$ radiolabelled DNA, $2 \mu \mathrm{l}$ of buffer $(10 \mathrm{~mm} \mathrm{Na}$ cacodylate, $\mathrm{pH}$ 7.0; Tris buffer must be avoided due to the presence of reactive amine functions) and $10 \mu \mathrm{l}$ of the drug at the desired concentration in the dark at room temperature, for $16 \mathrm{~h}$, prior to adding $5 \mu \mathrm{l}$ of a $50 \%$ glycerol solution containing tracking dyes. DNA samples were resolved by electrophoresis under non-denaturing conditions in $6 \%$ acrylamide gels for about $5 \mathrm{~h}$ at $300 \mathrm{~V}$ at room temperature in TBE buffer $(89 \mathrm{~mm}$ boric acid, $2.5 \mathrm{mM} \mathrm{Na} \mathrm{N}_{2}$ EDTA, $\mathrm{pH} 8.3$ ). Gels were transferred to Whatman 3MM paper, dried under vacuum at $80^{\circ} \mathrm{C}$, and then analyzed on a phosphorimager (Molecular Dynamics 445SI).

\section{References}

1) Hughes G. K., Lahey F. N., Price J. R., Nature (London), 162, 223224 (1948).

2) Macdonald P. L., Robertson A. V., Aust. J. Chem., 19, 275-281 (1966). 
3) Tillequin F., Michel S., Skaltsounis A.-L., "Alkaloids: Chemical and Biological Perspectives," Vol. 12, ed. by Pelletier S. W., Elsevier, New York, 1998, pp. 1-102.

4) Svoboda G. H., Lloydia, 29, 206-224 (1966).

5) Svoboda G. H., Poore G. A., Simpson P. J., Boder G. B., J. Pharm. Sci., 55, 758-768 (1966).

6) Scarffe J. H., Beaumont A. R., Gowther D., Cancer Treat. Rep., 67, 93-94 (1983).

7) Brum-Bousquet M., Mitaku S., Skaltsounis A.-L., Tillequin F., Koch M., Planta Med., 54, 470-471 (1988).

8) Elomri A., Mitaku S., Michel S., Skaltsounis A.-L., Tillequin F., Koch M., Pierré A., Guilbaud N., Léonce S., Kraus-Berthier L., Rolland Y., Atassi Gh., J. Med. Chem., 39, 4762-4766 (1996).

9) Costes N., Le Deit H., Michel S., Tillequin F., Koch M., Pfeiffer B., Renard P., Léonce S., Guilbaud N., Kraus-Berthier L., Pierré A., Atassi Gh., J. Med. Chem., 43, 2395-2402 (2000).

10) Guilbaud N., Léonce S., Tillequin F., Koch M., Hickman J. A., Pierré A., Anti Cancer Drugs, 13, 445-449 (2002).

11) David-Cordonnier M.-H., Laine W., Lansiaux A., Kouach M., Briand G., Pierré A., Hickman J. A., Bailly C., Biochemistry, 41, 9911—9920 (2002).

12) Doan Thi Mai H., Gaslonde T., Michel S., Tillequin F., Koch M.,
Bongui J.-B., Elomri A., Seguin E., Pfeiffer B., Renard P., David-Cordonnier M.-H., Laine W., Bailly C., Kraus-Berthier L., Léonce S., Hickman J. A., Pierré A., J. Med. Chem., 46, 3072-3082 (2003).

13) David-Cordonnier M.-H., Laine W., Joubert A., Tardy C., Goossens J.F., Kouach M., Briand G., Doan Thi Mai H., Michel S., Tillequin F., Koch M., Léonce S., Pierré A., Bailly C., Eur. J. Biochem., 270, 2848-2859 (2003).

14) David-Cordonnier M.-H., Laine W., Kouach M., Briand G., Vezin H., Gaslonde T., Michel S., Doan Thi Mai H., Tillequin F., Koch M., Léonce S., Pierré A., Bailly C., Bioorg. Med. Chem., 12, 23-29 (2004).

15) Michel S., Seguin E., Tillequin F., Curr. Med. Chem., 9, 1689-1700 (2002).

16) Shieh H. L., Pezzuto G. M., Cordell G. A., Chem.-Biol. Interact., 81, 35-55 (1992).

17) Elomri A., Skaltsounis A.-L., Michel S., Tillequin F., Koch M., Rolland Y., Pierré A., Atassi Gh., Chem. Pharm. Bull., 44, 2165-2168 (1996).

18) Pierré A., Kraus-Berthier L., Atassi Gh., Cros S., Poupon M. F., Lavielle G., Berlion M., Bizarri J. P., Cancer Res., 51, 2312-2318 (1991). 